\title{
Understanding agoraphobic avoidance: the development of the Oxford Cognitions and Defences Questionnaire (O-CDQ)
}

\author{
Laina Rosebrock ${ }^{1,2,3, *}$ (D) Sinéad Lambe ${ }^{1,2,3}$ (D) , Sophie Mulhall ${ }^{1,2}$, Ariane Petit ${ }^{1,2,3}$, Bao S Loe , $^{4}$ \\ Simone Saidel ${ }^{1,2}$, Maryam Pervez ${ }^{5}$, Joanna Mitchell ${ }^{1,2}$, Nisha Chauhan ${ }^{6}$, Eloise Prouten ${ }^{7}$, \\ Cindy Chan $^{6}$, Charlotte Aynsworth ${ }^{5}$, Elizabeth Murphy ${ }^{6}$, Julia Jones ${ }^{7}$, Rosie Powling 8 , \\ Kate Chapman ${ }^{8}$, Robert Dudley ${ }^{5,9}$, Anthony Morrison ${ }^{6,10}$, Eileen O’Regan ${ }^{7}$, David M Clark ${ }^{2,3,11}$, \\ Felicity Waite $^{1,2,3}$ (D) and Daniel Freeman ${ }^{1,2,3}$ (D)
}

${ }^{1}$ Department of Psychiatry, University of Oxford, Oxford, UK, ${ }^{2}$ Oxford Health NHS Foundation Trust, Oxford, UK, ${ }^{3}$ NIHR Oxford Health Biomedical Research Centre, Oxford, UK, ${ }^{4}$ The Psychometrics Centre, University of Cambridge, Cambridge, UK, ${ }^{5}$ Cumbria, Northumberland, Tyne and Wear NHS Foundation Trust, Newcastle upon Tyne, UK, ${ }^{6}$ Greater Manchester Mental Health Foundation Trust, Manchester, UK, ${ }^{7}$ Nottinghamshire Healthcare NHS Foundation Trust, Nottingham, UK, ${ }^{8}$ Avon and Wiltshire Mental Health Partnership (AWP) NHS Trust, Bath, UK, ${ }^{9}$ University of Newcastle, Newcastle upon Tyne, UK, ${ }^{10}$ Division of Psychology and Mental Health, University of Manchester, Manchester, UK and ${ }^{11}$ Department of Experimental Psychology, University of Oxford, Oxford, UK

${ }^{\star}$ Corresponding author. Email: laina.rosebrock@psych.ox.ac.uk

(Received 21 June 2021; revised 9 December 2021; accepted 14 December 2021; first published online 15 February 2022)

\begin{abstract}
Background: Many patients with mental health disorders become increasingly isolated at home due to anxiety about going outside. A cognitive perspective on this difficulty is that threat cognitions lead to the safety-seeking behavioural response of agoraphobic avoidance.

Aims: We sought to develop a brief questionnaire, suitable for research and clinical practice, to assess a wide range of cognitions likely to lead to agoraphobic avoidance. We also included two additional subscales assessing two types of safety-seeking defensive responses: anxious avoidance and within-situation safety behaviours.

Method: 198 patients with psychosis and agoraphobic avoidance and 1947 non-clinical individuals completed the item pool and measures of agoraphobic avoidance, generalised anxiety, social anxiety, depression and paranoia. Factor analyses were used to derive the Oxford Cognitions and Defences Questionnaire (O-CDQ).

Results: The O-CDQ consists of three subscales: threat cognitions (14 items), anxious avoidance (11 items), and within-situation safety behaviours (8 items). Separate confirmatory factor analyses demonstrated a good model fit for all subscales. The cognitions subscale was significantly associated with agoraphobic avoidance $(r=.672, p<.001)$, social anxiety $(r=.617, p<.001)$, generalized anxiety $(r=.746, p<.001)$, depression $(r=.619, p<.001)$ and paranoia $(r=.655, p<.001)$. Additionally, both the O-CDQ avoidance $(r=.867, p<.001)$ and within-situation safety behaviours $(r=.757, p<.001)$ subscales were highly correlated with agoraphobic avoidance. The O-CDQ demonstrated excellent internal consistency (cognitions Cronbach's alpha $=.93$, avoidance Cronbach's alpha $=.94$, within-situation Cronbach's alpha $=.93$ ) and test-re-test reliability (cognitions ICC $=$ 0.88 , avoidance ICC $=0.92$, within-situation ICC $=0.89$ ).

Conclusions: The O-CDQ, consisting of three separate scales, has excellent psychometric properties and may prove a helpful tool for understanding agoraphobic avoidance across mental health disorders.
\end{abstract}

Keywords: agoraphobic avoidance; cognitive model; measure development; safety-seeking behaviours; threat cognitions

(c) The Author(s), 2022. Published by Cambridge University Press on behalf of the British Association for Behavioural and Cognitive Psychotherapies. This is an Open Access article, distributed under the terms of the Creative Commons Attribution licence (https:// creativecommons.org/licenses/by/4.0/), which permits unrestricted re-use, distribution, and reproduction in any medium, provided the original work is properly cited. 


\section{Introduction}

The anxious avoidance of everyday situations such as public transport, shops or crowds agoraphobia - occurs across many different mental health disorders. Agoraphobia symptoms are a common feature in depression (Kennedy et al., 2007), social anxiety (Chambless, 1985), panic disorder (Clark, 1986) and psychosis (Freeman et al., 2019b). A survey of 1800 patients with psychosis attending mental health services indicated that nearly two-thirds were experiencing avoidance at agoraphobia levels (Freeman et al., 2019b). This anxious withdrawal often persists even after the remittance of the defining symptoms of a disorder (e.g. Davidson et al., 2016). A cognitive perspective is that the avoidance is a safety-seeking response to threat cognitions (i.e. anticipated harm). Existing self-report measures of threat cognitions have typically been developed for an individual disorder, leading to a sole focus on content characteristic of that condition. The diagnosis of agoraphobia has been particularly linked to threat cognitions of being trapped or having a panic attack. However, anxious avoidance is likely to occur in response to a wider range of threat cognitions. In depression, avoidance of everyday situations could be due to fears of failing or being rejected (e.g. Beck, 1967); in social anxiety, due to fears of being negatively evaluated or judged (e.g. Hofmann, 2007); and in psychosis, caused by fears of being attacked (Freeman, 2016) or being unable to cope with voices (Thomas et al., 2015). Although certain cognitions may be characteristic of particular disorders, they are not confined to those presentations. Our aim was to develop a single brief measure capturing key cognitions that feature in many clinical disorders which may drive agoraphobic avoidance.

Avoidance can be viewed as a safety-seeking behaviour (Salkovskis, 1991; Salkovskis et al., 1999), an action used to prevent a feared outcome from occurring that maintains the threat cognition via the prevention of the receipt of disconfirmatory evidence. To avoid confusion in clinical work, our group calls safety-seeking behaviours 'defences' (Freeman, 2016), as in the treatment of paranoia we encourage the learning of safety. When the feared outcome does not occur, individuals attribute this to the success of the safety-seeking or defence action rather than that the threat cognition was incorrect. Even when situations are entered, most anxious individuals use subtle within-situation safety-seeking behaviours. For example, individuals may try to complete the task as quickly as possible (i.e. rush), increase vigilance (i.e. focus on signs of danger in the environment or identify easy exits), or limit the likelihood of a social interaction (e.g. avoid eye contact or keep distance from others). These within-situation safety-seeking behaviours are similarly used across clinical diagnoses, including depression (e.g. Newby and Moulds, 2010), anxiety disorders (Salkovskis, 1991; McManus et al., 2008) and psychosis (Freeman et al., 2001).

The aim of this study was therefore to develop a brief measure, with three separate scales, assessing (1) key cognitions that may drive agoraphobic avoidance, (2) anxious avoidance of common everyday situations, and (3) within-situation safety-seeking behaviours. Importantly, the measure was designed to assess a broad range of threat cognitions so that it can be used across clinical disorders.

\section{Method}

\section{Participants}

We recruited three participant groups: non-clinical individuals $(n=612)$ scoring above the Mobility Inventory (MI) threshold for the presence of agoraphobia ( $\geq 2.3$ ); non-clinical individuals $(n=1335)$ scoring below the MI threshold for the presence of agoraphobia; and patients with psychosis $(n=198)$ being treated for agoraphobic avoidance as part of a clinical trial. See Table 1 for a summary of demographic characteristics.

The patients with psychosis were recruited as part of gameChange, a multi-site randomised controlled trial of an automated virtual reality intervention to reduce agoraphobic avoidance 
Table 1. Participant demographic and clinical characteristics

\begin{tabular}{lccc}
\hline & $\begin{array}{c}\text { Patients with psychosis } \\
(n=198)\end{array}$ & $\begin{array}{c}\text { Low agoraphobia } \\
(n=1335)\end{array}$ & $\begin{array}{c}\text { High agoraphobia } \\
(n=612)\end{array}$ \\
\hline Age (mean, SD) & $36.51(12.77)$ & $48.54(15.79)$ & $44.13(15.09)$ \\
Age range & $16-71$ & $18-84$ & $18-80$ \\
Gender & $n(\%)$ & $n(\%)$ & $n(\%)$ \\
Male & $136(68.7 \%)$ & $341(25.5 \%)$ & $136(22.2 \%)$ \\
Female & $61(30.8 \%)$ & $976(73.1 \%)$ & $458(74.8 \%)$ \\
Non-binary/other & 0 & $16(1.2 \%)$ & $16(2.6 \%)$ \\
Prefer not to say & 0 & $2(0.1 \%)$ & $2(0.3 \%)$ \\
Ethnicity & $171(86.4 \%)$ & $1269(95.1 \%)$ & $580(94.8 \%)$ \\
White & $4(2 \%)$ & $17(1.3 \%)$ & $8(1.3 \%)$ \\
Asian (any) & $4(2 \%)$ & $8(0.6 \%)$ & $1(0.2 \%)$ \\
Black (any) & $18(9.1 \%)$ & $28(2.1 \%)$ & $17(2.6 \%)$ \\
Mixed/multiple/other & 0 & $12(0.9 \%)$ & $6(1.0 \%)$ \\
Prefer not to say & & & - \\
Diagnosis & $80(41.0 \%)$ & - & - \\
Schizophrenia & $79(40.0 \%)$ & - & - \\
Psychosis not otherwise specified & $11(5.6 \%)$ & - & - \\
Schizoaffective disorder & $6(3.1 \%)$ & - & - \\
Bipolar affective disorder & $18(9.2 \%)$ & - & - \\
Depression with psychotic features & $2(1.0 \%)$ & - \\
Delusional disorder & $25.62(10.67)$ & & - \\
Age in first contact with services (mean, SD) & & -
\end{tabular}

(Freeman et al., 2019c). The main inclusion criteria for the gameChange trial are: (1) age 16 years + , (2) a diagnosis of non-affective or affective psychosis, and (3) self-reported difficulties going into everyday situations due to anxiety.

Non-clinical participants were recruited using advertisements on social media. The inclusion criteria were: 18 years or older and residing in the United Kingdom.

\section{Measures}

\section{Oxford Cognitions and Defences Questionnaire (O-CDQ; Supplementary Appendix 1)}

An item pool of 46 items was developed (see Table 2), corresponding to three distinct subscales (threat cognitions, anxious avoidance, and within-situation safety behaviours). The item pool consisted of 14 threat cognition items, 22 anxious avoidance items, and 10 within-situation safety behaviours (i.e. defences). The threat cognition items were chosen to correspond to seven different symptoms (depression, social anxiety, panic disorder, persecutory ideation, ideas of reference, aggression, and verbal hallucinations), with two items per presentation. The anxious avoidance and within-situation safety behaviour items included common places that people with agoraphobia typically avoid (e.g. cafés, shops) and strategies used to reduce anxiety in those situations (e.g. avoiding eye contact), respectively. The items were generated by four cognitive clinical psychologists using these guiding principles, a review of existing measures for several individual mental health disorders, and an analysis of responses in previous studies from patients with psychosis, Participants were asked to rate for the past fortnight how often they had a specific cognition, how frequently they had avoided situations, and how often they had used within-situation safety behaviours. Each item was rated on a 4-point scale from 0 (never) to 3 (always), with higher ratings indicating greater severity.

\section{Mobility Inventory for Agoraphobia (MI; Chambless et al., 1985)}

The MI is a self-report measure assessing current avoidance of situations due to anxiety (i.e. agoraphobia) when alone. Items ask about avoidance of places (e.g. restaurants, car parks), 
Table 2. Oxford Cognitions and Defences Questionnaire (O-CDQ) original item pool

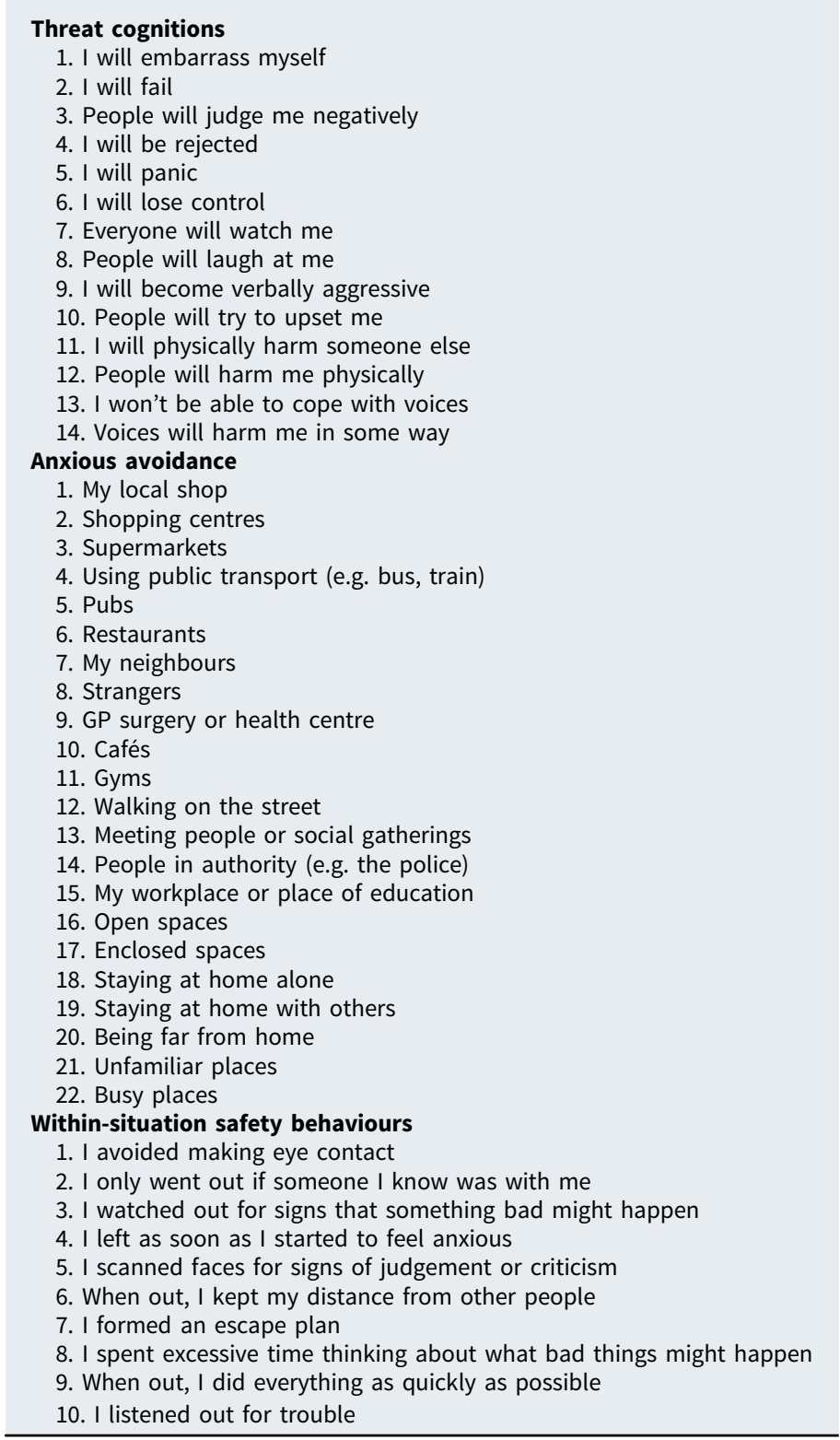

transportation (e.g. buses, trains), riding/driving in a car, and specific situations (e.g. walking on the street, standing in queues). Each item is rated on a 5 -point scale, from 1 (never avoid) to 5 (always avoid) and there is an option to select 'not applicable' (n/a). The average score is derived from all items that were not answered ' $\mathrm{n} / \mathrm{a}$ '. Higher average scores indicate higher levels of agoraphobia. An average score of 2.3 and above is considered the clinical cut-off (National Collaborating Centre for Mental Health, 2020). 
Oxford Agoraphobic Avoidance Scale (O-AS; Lambe et al., 2021)

The O-AS is a new 8-item scale assessing agoraphobic avoidance and anxiety in everyday situations. Items concern a range of everyday activities (e.g. travelling on a bus for several stops, sitting in a café for 10 minutes). Participants are asked to provide two ratings: whether they think they could do the task now (avoidance rating) and how anxious they would feel completing the task (distress rating). Higher scores on both the avoidance and distress scale indicate greater difficulties.

\section{Generalised Anxiety Disorder Scale-7 (GAD-7; Spitzer et al., 2006)}

The GAD-7 is a 7-item scale assessing symptoms of generalised anxiety in the past two weeks. Example items include 'feeling nervous, anxious or on edge' and 'feeling afraid as if something awful might happen'. Each item is rated on a 4-point scale, from 0 (not at all) to 3 (nearly every day). Higher scores indicate higher levels of generalised anxiety.

\section{Brief Fear of Negative Evaluation Scale (BFNE; Leary, 1983)}

The BFNE is a 12-item scale assessing fear of being negatively evaluated. Example items include 'I am usually worried about what kind of impression I make' and 'I am afraid that people will find fault with me'. Items are rated on a 5-point scale, from 1 (not at all characteristic of me) to 5 (extremely characteristic of me). Four items are reverse-coded. Higher scores indicate higher levels of concerns with being negatively evaluated by others.

\section{Patient Health Questionnaire-9 (PHQ-9; Kroenke et al., 2001)}

The PHQ-9 is a 9-item self-report questionnaire measuring symptoms of depression over the past two weeks (e.g. feeling down; difficulty concentrating; psychomotor retardation/agitation). Each of the items corresponds to a diagnostic criteria item for major depression. Items are rated on a 4-point scale, from 0 (not at all) to 3 (nearly every day). Higher scores indicate higher levels of depression.

\section{Revised Green et al. Paranoid Thoughts Scale (R-GPTS; Freeman et al., 2019a)}

The R-GPTS is an 18-item self-report questionnaire assessing paranoid thinking over the past two weeks. It contains two subscales: ideas of reference (10 items) and persecution ( 8 items). Example items for each subscale include 'I spent time thinking about friends gossiping about me' and 'I was sure someone wanted to hurt me'. Each item is rated on a 5-point scale, from 0 (not at all) to 4 (totally). Higher scores indicate higher levels of paranoid thinking.

\section{Procedure}

All participants completed the O-CDQ item pool, MI, R-GPTS and PHQ-9. The non-clinical participants completed the GAD-7 and the BFNE. Patients with psychosis also completed the O-AS.

Non-clinical participants completed all measures online. Patients with psychosis completed the measures either in person with the support of a research assistant, online, via post, or over the telephone (remote completion was implemented for participants after September 2020 due to the COVID-19 pandemic).

To evaluate test-re-test reliability, 372 non-clinical participants repeated the O-CDQ after two weeks. 


\section{Statistical analysis}

Analyses were conducted in R, version 4.0.3 (R Core Team, 2020) and SPSS, version 26.0 (IBM, 2020). Exploratory and confirmatory factor analyses (EFA and CFA) were used to derive the final set of items for the O-CDQ. CFA was conducted using the 'lavaan' package (Rosseel, 2012). We divided the participants into two groups: a derivation sample and a validation sample. The derivation sample included $75 \%$ of the combined group of non-clinical individuals scoring above the MI threshold for presence of agoraphobia (score $\geq 2.3$ ) and patients with psychosis. This consisted of 464 non-clinical participants with high levels of agoraphobia and 143 patients with psychosis (combined $n=607$ ). EFA was conducted with the derivation sample. The validation sample included the remaining $25 \%$ of the combined group of nonclinical individuals scoring above the MI threshold for presence of agoraphobia and patients with psychosis, and all non-clinical individuals scoring below the MI threshold for presence of agoraphobia. This consisted of 148 non-clinical individuals with high levels of agoraphobia, 55 patients with psychosis, and 1335 non-clinical individuals with low levels of agoraphobia (combined $n=1538$ ). CFA was conducted with the validation sample.

Given the small number of items for the threat cognitions subscale and the pre-specified theoretically driven factor structure, for this subscale only a CFA in the validation sample was run initially (using the 7-factor model with two items per factor) with the variance of all factors fixed to 1 for identification reasons. Although preferable to have more than two items per factor, it is possible to retain factors with two items if the factor loadings are high (above 0.70) and the between-factor correlations are moderate (e.g. Worthington and Whittaker, 2006; Yong and Pearce, 2013). Additionally, we examined other test theory methods (e.g. internal consistency and test-re-test reliability) to further support the use of the model.

Separate EFAs were conducted prior to CFA for the other two subscales (anxious avoidance and within-situation safety behaviours). Items that were either highly correlated $(r \geq 0.8)$ or had a low correlation with other items (did not have correlations above 0.3 with any other item) were deleted prior to EFA. EFA was estimated using principal axis factoring and oblique rotation, as we expected the factors to correlate. Parallel analysis and inspection of scree plots were used to determine the number of factors to extract, following the criteria of retaining only factors with eigenvalues above 1 . Items were considered for deletion based on factor loadings (did not load onto any factor above 0.30 , or had cross-loadings above 0.30 on multiple factors), communalities $(<0.30)$ and content (e.g. theoretically inconsistent or redundant). Once a final set of items was derived via EFA, CFA was conducted for the avoidance and withinsituation behaviours subscales separately to assess the model fit in the validation sample. The MLR robust maximum likelihood estimator was used to account for skewness of the data. Good model fit was determined using $\mathrm{Hu}$ and Bentler's (1999) recommended thresholds of above 0.95 on the comparative fit index (CFI) and Tucker-Lewis index (TLI), below 0.10 on the root mean square of approximation (RMSEA), and below 0.08 on the standardized root mean square residual (SRMR).

Pearson correlations were used to examine convergent validity. One-way ANOVAs were used to examine differences in cognitions, avoidance, and within-situation behaviours between the psychosis group, non-clinical individuals with agoraphobic avoidance, and non-clinical individuals without agoraphobic avoidance. Test-re-test reliability was run using the 'irr' package in R.

\section{Results}

\section{Threat cognitions subscale}

The initial CFA of the 14-item, 7-factor model (with two items per factor) indicated an excellent model fit $\left(\chi^{2}=295.93\right.$, d.f. $\left.=56, p<.001, \mathrm{CFI}=.970, \mathrm{TLI}=.951, \mathrm{RMSEA}=.053, \mathrm{SRMR}=.031\right)$ 
Table 3. Final items and factor loadings from exploratory factor analysis (EFA) and confirmatory factor analysis (CFA)

\begin{tabular}{|c|c|c|}
\hline Threat cognitions & & CFA loadings \\
\hline \multicolumn{3}{|l|}{ Depression } \\
\hline 1. I will embarrass myself & & 0.812 \\
\hline 2. I will fail & & 0.842 \\
\hline \multicolumn{3}{|l|}{ Social anxiety } \\
\hline 3. People will judge me negatively & & 0.913 \\
\hline 4. I will be rejected & & 0.842 \\
\hline \multicolumn{3}{|l|}{ Panic } \\
\hline 5. I will panic & & 0.857 \\
\hline 6. I will lose control & & 0.846 \\
\hline \multicolumn{3}{|l|}{ Social reference } \\
\hline 7. Everyone will watch me & & 0.881 \\
\hline 8. People will laugh at me & & 0.886 \\
\hline \multicolumn{3}{|l|}{ Harming others } \\
\hline 9. I will become verbally aggressive & & 0.774 \\
\hline 11. I will physically harm someone else & & 0.693 \\
\hline \multicolumn{3}{|l|}{ Persecution } \\
\hline 10. People will try to upset me & & 0.759 \\
\hline 12. People will harm me physically & & 0.651 \\
\hline \multicolumn{3}{|l|}{ Voices } \\
\hline 13. I won't be able to cope with voices & & 0.896 \\
\hline 14. Voices will harm me in some way & & 0.826 \\
\hline Anxious avoidance & EFA loadings & CFA loadings \\
\hline \multicolumn{3}{|l|}{ Shopping/being around others } \\
\hline 1. My local shop & 0.573 & 0.768 \\
\hline 2. Shopping centres & 0.851 & 0.866 \\
\hline 3. Supermarkets & 0.877 & 0.871 \\
\hline 4. Using public transport (e.g. bus, train) & 0.541 & 0.739 \\
\hline 12. Walking on the street & 0.510 & 0.718 \\
\hline \multicolumn{3}{|l|}{ Social places/meeting others } \\
\hline 7. My neighbours & 0.655 & 0.688 \\
\hline 9. GP surgery or health centre & 0.705 & 0.648 \\
\hline 10. Cafés & 0.480 & 0.794 \\
\hline 13. Meeting people or social gatherings & 0.549 & 0.757 \\
\hline 14. People in authority (e.g. the police) & 0.696 & 0.675 \\
\hline 15. My workplace or place of education & 0.487 & 0.649 \\
\hline Within-situation safety behaviours & EFA loadings & CFA loadings \\
\hline \multicolumn{3}{|l|}{ Avoiding others } \\
\hline 1. I avoided making eye contact & 0.539 & 0.680 \\
\hline 4. I left as soon as I started to feel anxious & 0.571 & 0.761 \\
\hline 6. When out, I kept my distance from other people & 0.796 & 0.849 \\
\hline 9. When out, I did everything as quickly as possible & 0.687 & 0.783 \\
\hline \multicolumn{3}{|l|}{ Hypervigilance } \\
\hline 3. I watched out for signs that something bad might happen & 0.891 & 0.854 \\
\hline 5. I scanned faces for signs of judgement or criticism & 0.413 & 0.726 \\
\hline 7. I formed an escape plan & 0.577 & 0.735 \\
\hline 10. I listened out for trouble & 0.777 & 0.862 \\
\hline
\end{tabular}

with almost all factor loadings above 0.70 (except for items 11 and 12, which were 0.69 and 0.65 , respectively). See Table 3 for factor loadings. Factor correlations are also provided in Supplementary Table 1. Furthermore, we conducted CFA examining a higher-order model to identify the existence of a global latent factor (i.e. threat cognitions). This indicated an acceptable model fit $\left(\chi^{2}=618.68\right.$, d.f. $=70, p<.001$, CFI $=.931$, TLI $=.910$, RMSEA $=$ .071 , SRMR $=.064)$. A total threat cognitions score, calculated by summing all the items, could therefore be used for subsequent validity analyses. Factor loadings for the higher-order model are provided in Supplementary Table 2. 
Cronbach's alphas indicated high levels of internal consistency for the total scale (.93) and all subscales: depression (.82), social anxiety (.88), panic (.86), social reference (.89), harming others (.67), persecution (.70) and voices (.87).

\section{Anxious avoidance subscale}

Factor analysis for the anxious avoidance subscale was appropriate as Bartlett's test of sphericity was significant $\left(\chi^{2}=5912.44\right.$, d.f. $\left.=231, p<.001\right)$ and the Kaiser-Myer-Olkin $(\mathrm{KMO})$ test of sampling adequacy was high $(\mathrm{KMO}=.92)$. No items were deleted prior to EFA. During EFA, 11 items were deleted: four items had communalities below 0.30 ('Enclosed spaces', 'Staying at home alone', 'Staying at home with others', 'Gyms'), five items were vaguely worded and/or did not fit with other items in their factor ('Busy places', 'Strangers', 'Being far from home', 'Unfamiliar places', 'Open spaces'), and two items were deleted due to very high factor loadings suggesting that they were driving the existence of that factor ('Restaurants', 'Pubs'). The scree plot and parallel analysis test of the remaining 11 items indicated that a two-factor model was the most appropriate fit for the data, with a factor correlation of 0.64. Factors were identified as 'Shopping/Being around others' and 'Social places/Meeting others'. This model explained $43.4 \%$ of the variance.

A CFA with the validation sample was conducted using the two-factor 11-item model from EFA. This indicated an excellent model fit $\left(\chi^{2}=218.15\right.$, d.f. $=43, p<.001$, CFI $=.961$, TLI $=.951$, RMSEA $=.051$, SRMR $=.030$ ). Factor loadings of the final items are presented in Table 3. The between-factor correlation was 0.886 . Additionally, a total anxious avoidance score, calculated by summing all the items, was used for subsequent validity analyses as a bi-factor model similarly indicated an excellent model fit $\left(\chi^{2}=112.74\right.$, d.f. $=33, p<.001, \mathrm{CFI}=.982$, TLI $=.971$, RMSEA $=$ .040 , SRMR $=.019$ ). Factor loadings for the bi-factor model are provided in Supplementary Table 3.

Cronbach's alpha indicated high internal consistency for the total scale (.94) and both subscales: shopping (.92) and social places (.88).

\section{Within-situation safety behaviours subscale}

Factor analysis for the within-situation safety behaviours subscale was appropriate as Bartlett's test of sphericity was significant $\left(\chi^{2}=2393.95\right.$, d.f. $\left.=34, p<.001\right)$ and the KMO test of sampling adequacy was high $(\mathrm{KMO}=.90)$. No items were deleted prior to EFA. During EFA, two items were deleted: one had communalities below 0.30 ('I only went out if someone I knew was with me'), and one was judged to be worded too vaguely ('I spent excessive time thinking about what bad things might happen'). The scree plot and parallel analysis test of the remaining eight items indicated that a two-factor model was the most appropriate fit for the data, with a factor correlation of 0.79 . Factors were identified as 'Self-protection' and 'Hypervigilance'. This model explained $46.5 \%$ of the variance.

A CFA with the validation sample was conducted using the two-factor, 8-item model from EFA. This indicated a good model fit $\left(\chi^{2}=193.69\right.$, d.f. $=19, p<.001$, CFI $=.959$, TLI $=$ .940 , RMSEA $=.077$, SRMR $=.036$ ). Factor loadings of the final items are presented in Table 3 . The between-factor correlation was 0.832 . As with the threat cognitions and anxious avoidance subscales, a total within-situation score, calculated by summing all the items, was used for subsequent validity analyses as a bi-factor model similarly indicated an excellent model fit $\left(\chi^{2}=92.24\right.$, d.f. $=12, p<.001$, CFI $=.981$, TLI $=.956$, RMSEA $=.066$, SRMR $=$ .019). Factor loadings for the bi-factor model are provided in Supplementary Table 4.

Cronbach's alpha indicated high internal consistency for the total scale (.93) and both subscales: avoiding others (.88) and hypervigilance (.88). 
Table 4. Mean scores and standard deviations for each O-CDQ subscale by participant group

\begin{tabular}{lc}
\hline O-CDQ subscale & Mean (SD) \\
\hline Threat cognitions total score & $19.19(8.76)^{\mathrm{a}}$ \\
Patients with psychosis $(n=198)$ & $18.84(8.38)^{\mathrm{a}}$ \\
High agoraphobia $(n=612)$ & $8.10(6.91)^{\mathrm{b}}$ \\
Low agoraphobia $(n=1335)$ & \\
Anxious avoidance total score & $18.07(8.56)^{\mathrm{a}}$ \\
Patients with psychosis $(n=198)$ & $15.28(7.18)^{\mathrm{b}}$ \\
High agoraphobia $(n=612)$ & $3.16(3.89)^{\mathrm{c}}$ \\
Low agoraphobia $(n=1335)$ & $14.59(5.42)^{\mathrm{a}}$ \\
Within-situation safety behaviours total score & $13.24(5.94)^{\mathrm{b}}$ \\
Patients with psychosis $(n=198)$ & $4.46(4.45)^{\mathrm{c}}$ \\
High agoraphobia $(n=612)$ & \\
Low agoraphobia $(n=1335)$ & \\
\hline
\end{tabular}

$\mathrm{a}>\mathrm{b}, \mathrm{b}>\mathrm{c}$ at $p<.01$.

\section{Validity}

The O-CDQ threat cognition subscale was significantly correlated with both the O-CDQ anxious avoidance $(r=.716, p<.001)$ and within-situation safety behaviours $(r=.799, p<.001)$ subscales.

\section{Correlations with agoraphobic avoidance}

The threat cognition subscale was significantly positively correlated with the MI $(r=.673$, $p<.001)$, the O-AS avoidance subscale $(r=.518, p<.001)$ and the O-AS distress subscale $(r=.676, p<.001)$. The O-CDQ anxious avoidance subscale was significantly positively correlated with agoraphobic avoidance as measured by both the MI $(r=.867, p<.001)$ and the O-AS avoidance $(r=.709, p<.001)$ and distress $(r=.822, p<.001)$ subscales. Lastly, the OCDQ within-situation safety behaviours subscale was positive correlated with agoraphobic avoidance measured by the MI $(r=.757, p<.001)$ and the O-AS avoidance $(r=.574, p<.001)$ and distress $(r=.752, p<.001)$ subscales.

\section{Correlations with other mental health outcomes}

Additionally, the O-CDQ threat cognition subscale was significantly positively correlated with the GAD-7 $(r=.746, p<.001)$, the BFNE $(r=.617, p<.001)$, the PHQ-9 $(r=.619, p<.001)$, the R-GPTS ideas of reference subscale $(r=.754, p<.001)$ and the R-GPTS persecutory ideation subscale $(r=.655, p<.001)$.

\section{Group differences for each subscale}

Mean scores for each of the three subscales for each group (non-clinical individuals without agoraphobic avoidance, non-clinical individuals with agoraphobic avoidance, and patients with psychosis) are given in Table 4. One-way ANOVAs indicated that there were significant differences between the non-clinical individuals and the patients with psychosis in levels of threat cognitions $\left(F_{2,2142}=520.301, p<.001\right)$, anxious avoidance $\left(F_{2,2142}=1356.02, p<.001\right)$ and within-situation safety behaviours $\left(F_{2,2142}=839.634, p<.001\right)$. Bonferroni-corrected multiple comparisons indicated that individuals without agoraphobic avoidance had the lowest scores on all subscales and non-clinical individuals with agoraphobic avoidance had lower scores than patients with psychosis for both the anxious avoidance and within-situation safety behaviours subscales but not the threat cognitions subscale. 


\section{Reliability}

Test-re-test reliability was excellent for all scales: threat cognitions $(n=372, \mathrm{ICC}=0.88,95 \% \mathrm{CI}$ : $0.85-0.90)$, anxious avoidance $(n=372$, ICC $=0.92,95 \%$ CI: $0.90-0.93)$ and within-situation safety behaviours $(n=372$ ICC $=0.89,95 \%$ CI: $0.87-0.91)$.

\section{Discussion}

A core tenet of cognitive theory is that cognitions drive unhelpful behavioural responses. This provides a powerful approach in understanding and treating many mental health disorders. One unhelpful response, agoraphobic avoidance, driven by fear cognitions, is a common feature across many mental health problems. This paper reports the development of the Oxford Cognitions and Defences Questionnaire (O-CDQ, Supplementary Appendix 1), designed for researchers and clinicians to quickly assess and understand the key cognitions and behaviours common to agoraphobic difficulties across clinical disorders. Factor analyses demonstrated an excellent model fit for the subscales assessing threat cognitions, anxious avoidance, and within-situation safety-seeking behaviours (i.e. defences). All subscales had high reliability. Each subscale consists of several factors but also comprises a general latent factor. Therefore, total scores for each subscale are used, making the scale easy to understand and administer. A total score from the three subscales is not meaningful and is not used. Assessed in a large participant group, the psychometric properties of the three scales are strong.

The three subscales of the O-CDQ - threat cognitions, anxious avoidance, and within-situation safety-seeking behaviours - were all highly correlated. This suggests that the measure is capturing the link between fearful cognitions (e.g. 'I will fail', 'I will embarrass myself, 'Everyone will watch me') and use of behaviours aimed at reducing the feared outcome from occurring. Importantly, the cognitions subscale was highly associated with two validated measures of agoraphobic avoidance. This subscale was also highly associated with measures of depression, social anxiety, generalized anxiety and paranoia. This suggests that this subscale may be useful in understanding key cognitions across these clinical presentations to inform formulation and link to interventions that directly target these cognitions. The avoidance and within-situation subscales were also highly correlated with existing measures of agoraphobic avoidance, supporting their validity. Furthermore, there were significant differences in scale scores between participants with and without agoraphobia symptoms.

There are several key limitations. The non-clinical participants were recruited online using social media and therefore will not be representative of the general population. The nonclinical participant group with high levels of agoraphobic avoidance was determined using a self-report scale rather than clinical interview. The patient group was limited to individuals with psychosis. Although patients with psychosis typically have high levels of anxiety and depression, future studies could test the scale in other diagnostic groups. Test-re-test data were only collected for the non-clinical group. Lastly, the cognitions subscale consisted of factors comprising two items. Although two-item factors are inferior to standard multi-item factors, the items were theoretically driven and the subscale demonstrated high internal consistency and test-re-test reliability, as well as excellent convergent validity. The O-CDQ is being used in the multi-centre gameChange clinical trial, and its true value will become apparent.

Supplementary material. To view supplementary material for this article, please visit: https://doi.org/10.1017/ S1352465822000030

\section{Acknowledgements. None}

Financial support. This work was funded by the National Institute for Health Research (NIHR) invention for innovation (i4i) programme (project II-C7-0117-20001). It was also supported by the NIHR Oxford Health Biomedical Research Centre (BRC). D.F. and D.C. are NIHR Senior Investigators. The views expressed are those of the authors and not necessarily 
those of the NHS, the NIHR or the Department of Health and Social Care. Felicity Waite is funded by a Wellcome Trust Clinical Doctoral Fellowship (102176/B/13/Z).

Conflicts of interest. The authors declare none.

Ethics statement. This study abided by the Ethical Principles of Psychologists and Code of Conduct. Ethical approval for the gameChange study was obtained from an NHS Research Ethics Committee (REC reference 19/SC/0075). Ethical approval for the collection of data from non-clinical participants was obtained from the Medical Sciences Inter-Divisional Research Ethics Committee (IDREC) at the University of Oxford (R63059/RE001).

Data availability statement. All requests for access to the de-identified data will be considered by the Chief Investigator of the study and team. The intent is to share data for reasonable requests. Data will be made available to external researchers subject to the constraints of the consent under which data were collected, with an appropriate data sharing agreement.

Author contributions. Laina Rosebrock: Conceptualization (lead), Formal analysis (lead), Writing - original draft (lead), Writing - review \& editing (equal); Sinead Lambe: Conceptualization (equal), Formal analysis (equal), Investigation (supporting), Writing - review \& editing (equal); Sophie Mulhall: Conceptualization (equal), Investigation (lead), Methodology (equal), Writing - review \& editing (equal); Ariane Petit: Writing - review \& editing (equal); Bao S. Loe: Formal analysis (supporting), Writing - review \& editing (equal); Simone Saidel: Investigation (supporting), Writing review \& editing (supporting); Maryam Pervez: Investigation (supporting), Writing - review \& editing (supporting); Joanna Mitchell: Investigation (supporting), Writing - review \& editing (supporting); Nisha Chauhan: Investigation (supporting), Writing - review \& editing (supporting); Eloise Prouten: Investigation (supporting), Writing - review \& editing (supporting); Charlotte Aynsworth: Investigation (supporting), Writing - review \& editing (supporting); Elizabeth Murphy: Investigation (supporting), Writing - review \& editing (supporting); Julia Jones: Investigation (supporting), Writing - review \& editing (supporting); Rosie Powling: Investigation (supporting), Writing - review \& editing (supporting); Kate Chapman: Project administration (equal), Supervision (supporting), Writing - review \& editing (supporting); Robert Dudley: Project administration (equal), Supervision (supporting), Writing - review \& editing (supporting); Anthony Morrison: Project administration (equal), Supervision (supporting), Writing - review \& editing (supporting); Eileen O’Regan: Project administration (equal), Supervision (supporting), Writing - review \& editing (supporting); David Clark: Conceptualization (equal), Funding acquisition (equal), Writing - review \& editing (supporting); Felicity Waite: Conceptualization (equal), Project administration (equal), Supervision (supporting), Writing - review \& editing (supporting); Daniel Freeman: Conceptualization (equal), Funding acquisition (lead), Investigation (equal), Project administration (lead), Writing - original draft (equal), Writing - review \& editing (equal).

\section{References}

Beck, A. T. (1967). Depression: Clinical, Experimental, and Theoretical Aspects. New York, USA: Harper \& Row.

Chambless, D. L. (1985). The relationship of severity of agoraphobia to associated psychopathology. Behaviour Research and Therapy, 23, 305-310. doi: 10.1016/0005-7967(85)90009-9

Chambless, D. L., Caputo, G. C., Jasin, S. E., Gracely, E. J., \& Williams, C. (1985). The mobility inventory for agoraphobia. Behaviour Research and Therapy, 23(1), 35-44. doi: 10.1016/0005-7967(85)90140-8

Clark, D. M. (1986). A cognitive approach to panic. Behaviour Research and Therapy, 24(4), 461-470. doi: 10.1016/00057967(86)90011-2

Davidson, S. K., Dowrick, C. F., \& Gunn, J. M. (2016). Impact of functional andstructural social relationships on two year depression outcomes: A multivariateanalysis. Journal of Affective Disorders, 193, 274-281. doi: 10.1016/j.jad.2015.12.025

Freeman, D. (2016). Persecutory delusions: a cognitive perspective on understanding and treatment. The Lancet Psychiatry, 3 , 685-692. doi: 10.1016/S2215-0366(16)00066-3

Freeman, D., Garety, P. A., \& Kuipers, E. (2001). Persecutory delusions: developing the understanding of belief maintenance and emotional distress. Psychological Medicine, 31, 1293-1306. doi: 10.1017/s003329170100455x

Freeman, D., Loe, B. S., Kingdon, D., Startup, H., Molodynski, A., Rosebrock, L., . . \& Bird, J. C. (2019a). The revised Green et al., Paranoid Thoughts Scale (R-GPTS): psychometric properties, severity ranges, and clinical cut-offs. Psychological Medicine, 51, 244-253. doi: 10.1017/S0033291719003155

Freeman, D., Taylor, K. M., Molodynski, A., \& Waite, F. (2019b). Treatable clinical intervention targets for patients with schizophrenia. Schizophrenia Research, 211, 44-50. doi: 10.1016/j.schres.2019.07.016

Freeman, D., Yu, L. M., Kabir, T., Martin, J., Craven, M., Leal, J., . . \& Waite, F. (2019c). Automated virtual reality (VR) cognitive therapy for patients with psychosis: study protocol for a single-blind parallel group randomised controlled trial (gameChange). BMJ Open, 9, e031606. doi: 10.1136/bmjopen-2019-031606

Hofmann, S. G. (2007). Cognitive factors that maintain social anxiety disorder: a comprehensive model and its treatment implications. Cognitive Behaviour Therapy, 36, 193-209. doi: 10.1080/16506070701421313 
Hu, L., \& Bentler, P. M. (1999). Fit indices in covariance structure modelling: sensitivity to underparameterized model misspecification. Psychological Methods, 3, 424-453. doi: 10.1047/1082-989X.3.4.424

IBM (2020). SPSS statistics version 26. Release 26.0.0. Armonk, NY, USA: IBM Corporation.

Kennedy, N., Foy, K., Sherazi, R., McDonough, M., \& McKeon, P. (2007). Long-term social functioning after depression treated by psychiatrists: a review. Bipolar Disorders, 9, 25-37. doi: 10.1111/j.1399-5618.2007.00326.x

Kroenke, K., Spitzer, R. L., \& Williams, J. B. (2001). The PHQ-9: validity of a brief depression severity measure. Journal of General Internal Medicine, 16, 606-613. doi: 10.1046/j.1525-1497.2001.016009606.x

Lambe, S., Bird, J. C., Loe, B. S., Rosebrock, L., Kabir, T., Petit, A., . . \& Freeman, D. (2021). The Oxford Agoraphobic Avoidance Scale. Psychological Medicine.

Leary, M.R. (1983). A brief version of the Fear of Negative Evaluation Scale. Personality and Social Psychology Bulletin, 9, 371-375. doi: 10.1177/0146167283093007

McManus, F., Sacadura, C., \& Clark, D. M. (2008). Why social anxiety persists: an experimental investigation of the role of safety behaviours as a maintaining factor. Journal of Behavior Therapy and Experimental Psychiatry, 39, 147-161. doi: 10. 1016/j.jbtep.2006.12.002

National Collaborating Centre for Mental Health (2020). The Improving Access to Psychological Therapies Manual. NHS England. https://www.england.nhs.uk/wp-content/uploads/2020/05/iapt-manual-v4.pdf

Newby, J. M., \& Moulds, M. L. (2010). Negative intrusive memories in depression: The role of maladaptive appraisals and safety behaviours. Journal of affective disorders, 126, 147-154. doi: 10.1016/j.jad.2010.03.012

R Core Team (2020). R: A Language and Environment for Statistical Computing. Vienna, Austria: R Foundation for Statistical Computing. Retrieved from: http://www.R-project.org/

Rosseel, Y. (2012). lavaan: an R package for structural equation modeling. Journal of Statistical Software, 48, 1-36. Retrieved from: http://www.jstatsoft.org/v48/i02/

Salkovskis, P. M. (1991). The importance of behaviour in the maintenance of anxiety and panic: a cognitive account. Behavioural and Cognitive Psychotherapy, 19, 6-19. doi: 10.1017/S0141347300011472

Salkovskis, P. M., Clark, D. M., Hackmann, A., Wells, A., \& Gelder, M. G. (1999). An experimental investigation of the role of safety-seeking behaviours in the maintenance of panic disorder with agoraphobia. Behaviour research and therapy, 37, 559-574. doi: 10.1016/s0005-7967(98)00153-3

Spitzer, R. L., Kroenke, K., Williams, J. B., \& Löwe, B. (2006). A brief measure for assessing generalized anxiety disorder: the GAD-7. Archives of Internal Medicine, 166, 1092-1097. doi: 10.1001/archinte.166.10.1092

Thomas, N., Farhall, J., \& Shawyer, F. (2015). Beliefs about voices and schemas about self and others in psychosis. Behavioural and Cognitive Psychotherapy, 43, 209-223. doi: 10.1017/S1352465813000817

Worthington, R. L., \& Whittaker, T. A. (2006). Scale development research: a content analysis and recommendations for best practices. The Counseling Psychologist, 34, 806-838. doi: 10.1177/0011000006288127

Yong, A. G., \& Pearce, S. (2013). A beginner's guide to factor analysis: focusing on exploratory factor analysis. Tutorials in Quantitative Methods for Psychology, 9, 79-94. doi: 10.20982/tqmp.09.2.p079

Cite this article: Rosebrock L, Lambe S, Mulhall S, Petit A, Loe BS, Saidel S, Pervez M, Mitchell J, Chauhan N, Prouten E, Chan C, Aynsworth C, Murphy E, Jones J, Powling R, Chapman K, Dudley R, Morrison A, O’Regan E, Clark DM, Waite F, and Freeman D (2022). Understanding agoraphobic avoidance: the development of the Oxford Cognitions and Defences Questionnaire (O-CDQ). Behavioural and Cognitive Psychotherapy 50, 257-268. https://doi.org/10.1017/S1352465822000030 